\title{
VIVENCIAS DE LAS MUJERES EGRESADAS DEL PROGRAMA DE POSGRADO EN TECNOLOGÍA Y SOCIEDAD (PPGTE) DE LA UNIVERSIDAD TECNOLÓGICA FEDERAL DEL PARANÁ (UTFPR)
}

\section{Experiences of women graduated from the Graduate Program in Technology and Society (PPGTE) of the Universidad Tecnológica Federal del Paraná (UTFPR)}

\section{Maria Sara de Lima Dias}

Profesora Adjunta del Departamento de Estudios Sociales, Programa de Posgrado en Tecnología y Sociedad, Maestría y Doctorado de la Universidad Tecnológica Federal de Paraná, Brasil Correo-e: mariadias@utfpr.edu.br https://orcid.org/0000-0001-7296-6400

\section{Paula Caldas Brognoli}

Becaria de Iniciación Científica en la Universidad

Tecnológica Federal de Paraná, Brasil

Correo-e: paulabrognoli@gmail.com orcid.org/0000-0003-3217-557X

Recibido: $27 / 7 / 2019 \bullet$ Aprobado: 7/2/2020

Cómo citar: de Lima Dias, M. S., \& Caldas Brognoli, P. (2020). Vivencias de las mujeres egresadas del Programa de Posgrado en Tecnología y Sociedad (PPGTE) de la Universidad Tecnológica Federal del Paraná (UTFPR). Ciencia y Sociedad, 45(1), 37-49. Dio: https://doi.org/10.22206/cys.2020.v45i1.pp37-49

\section{Resumen}

Esta investigación tuvo como objetivo evaluar si aspectos de género interfieren en las trayectorias académicas. El referencial teórico-epistemológico del estudio ha sido el histórico-cultural, para analizar distinciones existentes para las mujeres en el ejercicio de actividades investigativas. El método de investigación cualitativo fue de particular relevancia para analizar si existía una disolución de viejas desigualdades sociales dentro de la diversidad del ambiente académico. Como instrumento de investigación se utilizó la entrevista abierta, realizada a cinco mujeres egresadas del programa, tres de la maestría y dos del doctorado. El análisis de las narrativas permitió constatar que la carrera académica es una de las pocas alternativas en que la mujer puede trabajar y estudiar al mismo tiempo. En contraste, la asunción de múltiples papeles sociales y el esfuerzo emprendido para conciliar jornadas dobles de trabajo, y la falta de apoyo familiar y financiero para los estudios son vistos como obstáculos para la formación. Asimismo, las

\begin{abstract}
The research was oriented to evaluate if aspects of the gender interfere in the academic trajectories. The theoretical-epistemological referential of the historical-cultural perspective guides this study to make it possible to analyze the existing distinctions for women in the exercise of investigative activities. The method of qualitative research is of particular relevance in analyzing whether there is the dissolution of old social inequalities within the diversity of the academic environment. As a research instrument, we used open interviews carried out with five women graduates of the program, three of the master's and two of the doctorate. The analysis of the narratives allowed us to state that the academic career is one of the few alternatives in which women can work and study at the same time. In contrast, the assumption of multiple social roles and the effort undertaken to reconcile double working hours and the lack of family and financial support for studies they were seen as obstacles to training. Likewise, the narratives made
\end{abstract}


narrativas permitieron visibilizar un conjunto de "barreras" para seguir la carrera científica, que se refieren a: doble jornada de trabajo, maternidad, productividad en investigación, competencia, prejuicio y discriminación de género; de esta manera se reafirma la necesidad de desarrollar políticas públicas para ampliar no solo el acceso, sino la permanencia de las mujeres en la universidad.

Palabras clave: formación; trabajo; género; tecnología; ciencia.

\section{Introducción}

El trabajo de la mujer, desde un punto de vista general, es abordado por teóricos de diferentes campos del conocimiento, quienes apuntan hacia un nítido contraste en cuanto a la trayectoria de la mujer en el mercado de trabajo. El bajo porcentaje estadístico de mujeres que ocupan posiciones del poder en cargos de liderazgo demuestra un proceso histórico y social de exclusión.

El problema en la carrera científica es explicitado en Brasil como un espacio de poder masculino. Al analizar la historia de la presidencia de la asociación "Sociedad Brasileña de Investigación Científica" (SBPC) encontramos solo a finales de los 80 a la primera científica elegida como presidente de la SBPC. La profesora Carolina Bori (1924-2004), a pesar de haber ingresado en la SBPC en 1969, fue elegida presidenta de la entidad veinte años después de su incorporación (Melo y Rodrigues, 2018). Así, el patriarcado en las ciencias ha alimentado algunas de las diferencias de género, sea restringiendo el acceso de las mujeres a la academia o dificultando puestos de liderazgo en las ciencias.

Los movimientos feministas y la lucha por la igualdad han captado algunos logros. En las últimas décadas la mujer ha tenido acceso a la enseñanza superior en las áreas de tecnologías y ciencias, en general. No obstante, el alto nivel de educación no it possible to make visible a set of "barriers" to follow the scientific career, which refer in the: double working hours, maternity, productivity in research, competence, prejudice and gender discrimination; thus reaffirming the need to develop public policies to expand not only access, but also the permanence of women in the university.

Keywords: Formation, job, genre, technology and science.

ha sido suficiente para aumentar la renta percibida, ni para resolver formas de discriminación de género. Recientemente, las mujeres con acceso a cursos de posgrado evidenciaron una mayor participación en el campo de la educación superior en Brasil. Sin embargo, poco se conoce sobre la trayectoria de la mujer y sus vivencias en la academia, siendo este un tema aún por aclarar.

La trayectoria académica y profesional está relacionada con la vivencia del individuo, forma su identidad y le permite hacer elecciones. Según Benevides Soares, de Almeida Santos, de Andrade, y Siqueira de Souza (2017), las expectativas académicas involucran aspectos cognitivos, motivacionales y afectivos traídos por los estudiantes e investidos en la formación. Según Ostrovski, Sousa y Raitz (2017) la elección profesional, por medio de las influencias intrínseca y extrínseca que la condicionan, es considerada un momento de transición, proceso que produce implicaciones en la identidad profesional. Estas relaciones permiten al individuo construir su trayectoria de acuerdo con la carrera que eligió. Según Closs y Rocha-de-Oliveira (2015), la eligibilidad de carrera no está relacionada solo con los sujetos, sino también con organizaciones y profesiones, así como las relaciones entre las mismas, con las personas y sus trayectorias profesionales.

Según Dias y Soares (2012), el proceso transitivo de la carrera se articula con sentidos personales y 
particulares de la elección inicial del curso superior. Por lo tanto, la apreciación de la futura carrera pasa por diferentes referencias que se originan de las experiencias en el contexto universitario al contexto familiar y al mundo del trabajo. González Rey (2005) afirma que la formación profesional es constitutiva de una determinada cultura y, en este sentido, la formación en el área de la ciencia y la tecnología es un aspecto peculiar de la formación de estas mujeres egresadas del programa.

El camino profesional en un programa de posgrado en Tecnología y Sociedad nos hace pensar sobre la inclusión de mujeres en área de investigación en ciencias y tecnologías. La elección profesional y la formación en la enseñanza universitaria ha sido uno de los temas debatidos en la contemporaneidad por autores como Santos y Marques (2015); Peñaloza, Diógenes y Sousa (2008); Madalozzo y Artes (2017) y Bardagi, Lassance, Paradiso y Menezes (2006). Para Santos y Marques (2015) la docencia en las escuelas de enfermería se convirtió en un atractivo para la mujer que deseaba estudiar y trabajar. En Peñaloza et al., (2008) al estudiar la inserción de la mujer en el mercado de trabajo y su acceso a la enseñanza universitaria se demuestra que, distinto del hombre, las cuestiones profesionales ocupan un espacio secundario en la vida de la mujer. Respecto a esta perspectiva estamos en desacuerdo, una vez que las elecciones profesionales femeninas son amplias y, más allá, sus ambiciones no están restringidas a un empleo formal, aunque buscan también el posgrado como una forma de mayor calificación.

Madalozzo y Artes (2017) afirman que las mujeres que trabajan en profesiones masculinas tienen una ganancia salarial similar a la de los hombres por su elección, solo disminuye la diferencia salarial con sus pares si la opción es por una profesión imperial (derecho, ingeniería o medicina). No estamos de acuerdo con las afirmaciones de los autores, ya que no existen profesiones masculinas, y la mujer puede ocupar diferentes puestos de trabajo. Incluso, esta es una de las reclamaciones y uno de los factores de movilización por la igualdad de género.

El carácter excluyente de la división social del trabajo es a menudo establecido por carreras en las que la mujer tiene un salario menor que el recibido por el hombre. Bardagi, Lassance, Paradiso y Menezes (2006) realizaron una investigación en la que se buscó estudiar la satisfacción con la elección profesional y las expectativas en cuanto al ingreso en el mundo laboral. Se observó que el descontento con el mercado de trabajo fue un factor importante de insatisfacción con la elección profesional, mientras que la identificación personal se mostró relacionada con la satisfacción. Así, mayor es la identificación de la mujer con la profesión, mayor es la satisfacción con la elección.

De acuerdo con Da Silva y Ribeiro (2014), la existencia de barreras para el acceso a niveles laborales de mayor jerarquía y prestigio compromete, generalmente, a las mujeres en la construcción de su carrera. Aunque la participación de las mujeres sea equitativa desde el punto de vista numérico, la jerarquía académica estará ocupada, sobre todo, por hombres, independientemente del área del conocimiento. Para hacer este escenario menos desigual, las mujeres necesitan una carrera profesional más cualificada.

La elección profesional de una trayectoria en la docencia implica la búsqueda de algún sentido personal para sus actividades. Según Antunes (2015), el sentido dado al trabajo en el mundo capitalista es completamente distinto del sentido asignado por la humanidad. De acuerdo con los datos de la Coordinación de Perfeccionamiento de Personal de Nivel Superior CAPES (2016) sobre el Sistema Nacional de Posgrado, las mujeres son la mayoría en ese nivel de la educación brasileña. Los números indican 165.564 mujeres matriculadas y tituladas 
en cursos de maestría y doctorado, mientras que los hombres suman 138.462 , una diferencia de aproximadamente el $19 \%$ a favor de las mujeres.

El objeto de estudio de esta investigación se dirige, específicamente, al programa de posgrado en Tecnología y Sociedad, el PPGTE, un programa con más de veinte años de existencia y que ha contribuido a la visibilidad de la mujer en el área. El programa congrega a docentes investigadores de diferentes áreas del conocimiento: letras, historia, sociología, ingeniería, física, matemáticas, psicología, educación, artes, diseño, filosofía, arquitectura, derecho, medio ambiente, en investigaciones que abarcan las interacciones entre Tecnología y Sociedad. El objetivo del programa es investigar las transformaciones causadas por las tecnologías y actividades realizadas por la sociedad en los campos material y cultural. En este sentido, interesa al programa verificar cómo las innovaciones tecnológicas interfieren en la vida de las personas, en su manera de trabajar, aprender, pensar, simbolizar y actuar en el mundo.

Aunque la participación femenina en el posgrado se esté incrementando, existen desafíos para una plena igualdad de géneros. La mayoría de la población brasileña es femenina y con más años de estudio que los hombres, a pesar de ello ganan menos; además, la igualdad económica entre sexos, en el ritmo actual, puede tardar 170 años, alerta el The Global Gender Report (2016). Cuestionar el perfil de la mujer en el posgrado en el campo de la ciencia, tecnología y sociedad (CTS) en Brasil es un tema de suma importancia, puesto que puede incentivar nuevas discusiones sobre el acceso y la permanencia de la mujer en el medio académico y traer luz a las políticas educativas de incentivo a la continuidad de los estudios en la enseñanza superior. Por ello, se estableció como objetivo describir las trayectorias de mujeres egresadas de un programa de posgrado de maestría y doctorado en Tecnología y Sociedad (PPGTE).

\section{El marco teórico}

La referencia del marco teórico-epistemológico desde la perspectiva histórico-cultural guía este estudio, cuyo propósito es describir el perfil y las trayectorias de las mujeres en un programa de maestría y doctorado en Tecnología y Sociedad (PPGTE). Programa que apunta a la formación y perfeccionamiento de los recursos humanos comprometidos con la producción interdisciplinar del conocimiento.

En la investigación sobre la constitución de sujetos se sobrepasan los procesos formativos que se desarrollan en los contextos educativos. Así, por ejemplo en la psicología lanzó las bases para una ciencia unificada del comportamiento humano (Vygotski, 2003, p. 10). Se intenta mostrar las consecuencias de un concepto de subjetividad comprometido con una representación teórica del objeto de estudio en su trayectoria socio-profesional. Para González Rey (2002) la epistemología cualitativa es un esfuerzo en la búsqueda de diferentes formas de producción de conocimiento en psicología, que permitan la creación teórica acerca de la realidad y la subjetividad humana.

Considerando que la experiencia del sujeto en relación con el mundo es siempre mediada por los horizontes sociales de valores, estos son, según Vygotski (2003), los mecanismos por los cuales la cultura se convierte en parte de la subjetividad. Ser profesora graduada, en este sentido, es la expresión objetiva de una realidad de posgrado subjetivado, González Rey (2005).

La epistemología cualitativa se basa en la idea de que el conocimiento es una producción constructiva e interpretativa de la realidad. En su carácter interpretativo, el proceso de producción de conocimiento enfatiza el significado de unicidad como un nivel legítimo de producción de conocimiento. Tratando de describir el perfil y las trayectorias de 
las mujeres graduadas en un programa de posgrado "la singularidad se constituye como una realidad diferente en la historia de la constitución subjetiva del individuo" (González Rey, 2002, p. 35). La familiarización con el contexto del curso de posgrado y con los estudiantes permitió identificar cuestiones fundamentales para la interpretación de la realidad formativa (Montero, 2012).

\section{Método, instrumento y procedimiento}

El tipo de investigación exploratoria (Gil, 2008) fue considerado como el más adecuado para la población en cuestión, orientado a un estudio de caso. De este modo, se hizo una invitación a las mujeres egresadas del programa y se les ofreció un plan para la realización de las entrevistas presenciales. Se realizaron entrevistas basadas en un cuestionario sobre datos generales y específicos de su formación. Se interrogó sobre si su trabajo actual se relaciona con el área de la educación; cuál es su percepción sobre los efectos del postgrado en la carrera docente; descripción de su trayectoria durante los estudios, como describe la relación con los profesores y colegas; y cuál es el significado de su trabajo y de la carrera. Las narraciones fueron grabadas y posteriormente transcritas. Para este artículo se consideraron las entrevistas realizadas a cinco mujeres, como un momento en que se alcanzó lo que para Montero (2012) se define como la saturación de los resultados, punto en el que la presencia de ciertos temas, de ciertas versiones, está claramente definido.

\section{Discusión de resultados}

Las egresadas tenían formaciones en diferentes áreas: economía (2), artes (1), derecho (2), administración (1). Las líneas de investigación: Mediaciones y Cultura, Tecnología y Desarrollo y Tecnología y Trabajo. De las entrevistadas dos (2) realizaron maestría, una (1), doctorado y dos (2) maestría y doctorado. A cada una de las entrevistadas se les atribuyó una letra: (D) si era doctora y (M) si era magíster. Asimismo, se les asignó un número del $1 \mathrm{al} 5$, en el sentido de protección de identidad de las participantes y para permitir la decodificación en el proceso de análisis. El proceso de análisis propone el recorte de elementos que son capaces de demostrar la importancia del contexto (formación en posgrado) y sus impactos en la singularidad y la subjetividad (narrativa de las mujeres).

La primera entrevistada (D-1) es una profesora universitaria, casada, con un hijo de tres años, quien cursó la maestría en el Departamento de Economía y el doctorado en el programa de postgrado del PPGTE. Tenía ganas de continuar sus estudios y vivir en otro país, pero por situaciones personales y por cuestiones de ayuda, como la falta de una beca universitaria, desistió, y en el ańo 2008 pasó a ser profesora en una universidad pública federal. Valentova Otta, Silva y McElligott (2017) observaron que hay una distribución desigual de becas para el género femenino, de un total de 4.859, solo 976 (20,08\%) son para investigación de las mujeres. En algunas áreas, las mujeres representan menos del $5 \%$ del total de investigadores. El testimonio, proporcionado por Valentova et al., (2017), que nos apoya sobre las dificultades de ayuda en el posgrado.

Un rasgo común lo constituye algún tipo de obstáculo vivido para el desarrollo de la carrera de la mujer en el área académica. Como en este fragmento de (D-1): "Terminé renunciando a salir al exterior". La ausencia de un recurso económico es un elemento que representa un obstáculo y que también involucra aspectos emocionales. El factor social y económico muchas veces no permite a la mujer alcanzar la realización de su deseo de continuar sus estudios en algún momento de su trayectoria. Algunos obstáculos para la formación ocurren en casi todas las entrevistadas. La dificultad para ocupar espacios y los obstáculos de no lograr la beca caracteriza una realidad de exclusión para las mujeres. Para Bolzani 
(2017), algunas alternativas son las premiaciones y homenajes de las agencias y gobiernos, esos reconocimientos serían capaces de aumentar la autoconfianza de las mujeres y serían un incentivo para la participación de más mujeres en el medio académico.

En este punto, la misma entrevistada demuestra las dificultades presentes en conciliar la condición social de ser mujer y los innumerables papeles sociales asumidos por ella: rol de madre, mujer e investigadora, como relata (D- 1): "Tengo un hijo, él tiene tres años, nació al final de mi doctorado, casi junto con la tesis". La expresión adquiere significación solo según el contexto y el lugar en que el sujeto se sitúa; para Vygotski (2003) los procesos humanos tienen su origen en las relaciones sociales, en este caso la relación con la tesis es también la relación con el hijo, en virtud del contexto.

La relación entre la vida pública, propia de la formación, y la vida privada o personal, también es una relación con la cultura universitaria; por lo que son múltiples las relaciones que deben ser investigadas (González Rey, 2005). Su expresión subjetiva es capaz de representar la dificultad de conciliar los roles (D-1): "Difícil, difícil, dificilísimo, así, creo, yo siento decir que para mí la maternidad fue un trauma, el verdadero trauma". Este momento de su trayectoria retrata los problemas de conciliar nuevamente el ámbito público y el privado, la vida académica con la maternidad. Para González Rey (2005) la identidad de profesora es afirmada en la forma de diferencia y de igualdad, conforme a los grupos sociales de los cuales forma parte; así, formar parte de la academia es pensar en un universo en que no existen hijos, o la maternidad no es bienvenida.

En otro tramo de su narrativa habla sobre los papeles atribuidos alrededor de carrera de la mujer, conforme (D-1): "atribuciones y tareas que te imponen...te adjudican..."
De este modo, los significados de la formación generan sentimientos a veces contradictorios, para (D-1): "Yo amo, me encanta la carrera de docente". Demuestra su satisfacción con la elección profesional; en oposición, en otro momento de su informe, presenta la insatisfacción con el doctorado: "No salió ningún artículo de mi tesis, no produjo nada, y nunca más lo cogí, nunca más lo miré". La trayectoria expresa sentimientos contradictorios, momentos de éxito en relación con su formación profesional y otros de frustración.

El carácter interactivo de la subjetividad muestra la importancia de las relaciones intersubjetivas, (D-1): "Siempre tuve mucho apoyo de mis padres para estudiar, mi madre también era profesora, y mi padre no, no tuvo mucha formación, pero siempre me animó". Es esto exactamente lo que le permitirá continuar en su trayectoria académica, puesto que son los apoyos y el incentivo de los padres los que le ayudará a alcanzar sus objetivos de carrera. Al tratar de la elección profesional demuestra la adhesión a su actividad profesional, configurando una identidad de profesora, (D-1): "Tengo mucho éxito con la economía. No me mudaría por nada, ni por un salario más alto". La trayectoria de la entrevistada está relacionada con la configuración de una identidad profesional que se constituye a partir del trabajo. (D-1): "Que lo bueno de ser profesor (...) Porque usted de alguna manera, usted los inspiró a seguir un cierto camino, (...) Creo que eso es lo más divertido en la carrera”. Hay un conjunto de elementos en esta trayectoria, aspectos familiares, sociales y el trabajo actual, que pueden caracterizar esta narrativa como una historia de éxito en la carrera.

La entrevistada (M-2) es una maestra en tecnología y sociedad, posee una unión estable, tiene una hija y actualmente trabaja en el Instituto Ciudad Júnior como directora de proyectos, es una de las fundadoras de la institución. Su trabajo es continuación de su 
investigación de maestría. En su narrativa relata la importancia del acceso al posgrado (M-2): "Mi trabajo es la continuación de mi investigación de maestría”. Según González Rey (2002), la mujer científica también es la investigadora, con una subjetividad que se afirma en una historia de vida que es constituida en el espacio de la formación. La vinculación de la entrevistada con otras personas en el posgrado demuestra la configuración de una subjetividad profesional que se desarrolla en su hacer, en su acción. De tal forma que va configurando en su narrativa una identidad de mujer profesional e investigadora: (M-2): "Fue muy buena, solo tengo buenas cosas para decir, solo tengo que agradecer por la oportunidad que me ha dado, me pongo emocionada al hablar". La importancia de la maestría se constituye subjetivamente como un proceso de representación de sí y del mundo a su alrededor, como expresa: "Acabé descubriendo un mundo". La entrada en el posgrado significó nuevos conocimientos y oportunidades (M-2): "Llevó esa investigación a tener un resultado muy positivo, y que ha llevado a la fundación de la institución en la que trabajo actualmente". Su narrativa también trae elementos para una interpretación de posibles obstáculos en su formación (M-2): "Yo no fui becaria, por eso participaba de todo lo que existía". Su trayectoria académica revela elementos que pueden contribuir a la mejora del programa (M-2): "Me gustaría haber tenido más clases de metodología de la investigación, pero esta era una demanda muy particular". La observación específica de su narrativa sobre el programa pasa por el área de la comunicación, que es un problema de su trayectoria de vida y no del programa, como describe (M-2): "Yo no tuve, así, una experiencia muy grande en el área de la comunicación de investigación". La ideología opera en los elementos de sentido, la relatada deficiencia en metodología guarda relación con la formación superior, a lo que podría ser una crítica al proceso académico, se constituye en un problema individual y continúa (M-2): "Me sentí en un problema, como en un desfase en el proceso, pero era una cosa mía, no de la estructura del programa". La expresión adquiere significación en el contexto, según el espacio apropiado, lugar determinado por el participante del programa. La entrevistada apunta al papel del profesor y al lugar del alumno en la formación académica, (M-2): "El profesor tiene papel de incentivador, motivador, es lo que da el impulso, el papel del profesor es de orientador". Los alcances de sus acciones describen una trayectoria de éxito personal y profesional, configurando una identidad de investigadora (M-2): "Yo conseguí trabajar las relaciones de poder dentro del proceso de aprendizaje en cuanto a la relación e interacción entre el profesor y el estudiante". El proceso de desarrollo del conocimiento se sintetiza en una expresión (M-2): "Aprendizaje fantástico".

La entrevistada (D-3) es profesora universitaria, graduada en Administración y Derecho, finalizó su maestría y regresó al programa de posgrado para asistir al doctorado, está casada, sin hijos y le gusta mucho trabajar con la educación. La subjetividad es un sistema complejo de significaciones y sentidos que se producen en la vida cultural humana (González Rey, 2005). En su narrativa, los elementos como edad, curso de formación inicial, trabajo, o situación civil, por ejemplo, se relacionan con aspectos que facilitan o dificultan la formación académica (D-3): "Siempre he trabajado como profesora del magisterio superior". La experiencia previa demuestra la situación social de su desarrollo, y la formación modifica no solo los modos de vida, sino las visiones de mundo de las egresadas. (D-3): "Uno de los temas de mi investigación era justamente la cuestión, la relación entre educación y trabajo". La elección del tema se caracteriza por variaciones estructurales en el discurso (D-3): "La educación viene permeando toda mi formación". La narración remite a la memoria y la historia profesional y evidencia la oportunidad que caracteriza el posgrado (D-3): "El PPGTE me surgió como 
una posibilidad, principalmente, por el carácter interdisciplinario que tenía y por mi interés en la línea de tecnología y trabajo". Los aspectos de la interdisciplinariedad del programa se enfatizan en su discurso como elementos facilitadores de la integración a la formación en el posgrado.

La formación profesional tiene un papel esencial en diferentes momentos de su vida y son narrados como lugar para ser y lugar para investigar (D-3): "En cierto modo la UTFPR también siempre estuvo en mis horizontes como espacio de trabajo e investigación". El lugar en que se tiene la posibilidad de transformarse es un lugar instituido, pero también un lugar subjetivamente apropiado (González Rey, 2005). Dotado, por lo tanto, de sentido, (D-3): "Yo trato el PPGTE como si él fuera una casa, sabe". La imagen idealizada de la casa representa en la subjetividad un sistema de sentidos y significados que aun siendo simbólicos actúan en el proceso de subjetivación, como en: "Yo fui becaria en la maestría y eso fue determinante para que pudiera dedicarme a los estudios y a la conducción de la maestría”. El análisis de las condiciones sociales es determinante para el acceso y la permanencia en el programa, las normas de acceso a la beca, por ejemplo, facilitan el desarrollo de una mayor justicia social e igualdad en el acceso a la formación.

En la existencia social del sujeto participan tantos elementos de la subjetividad social (González Rey, 2002) como de la subjetividad individual; así en los juegos de comunicación que se establecen entre el entrevistador y los entrevistados aparecen en la forma en que el entrevistado se expresa (D-3): "Podría haber producido más". No se trata de estandarizar las diferencias entre las configuraciones subjetivas presentadas, ni tampoco cuantificar patrones de respuesta humana, sino diferenciar las expresiones que pueden representar no fallas, sino puntos a mejorar en el programa (D-3): "He encontrado las disciplinas siempre ofrecidas, de una forma muy, muy adecuada, muy alineada a mis demandas". Relata la satisfacción por el programa y se observa que algunas de las experiencias vivenciadas están profundamente arraigadas en su historia profesional y personal, integrando la formación de la persona, su experiencia de vida, su patrimonio vivido, como en este relato (D-3): "He tenido muchas dificultades en establecer esos equilibrios, desde la maestría he buscado mucho auxilio de la psicoterapia para mantener ese proceso". La subjetividad individual se va afirmando en la narrativa al traer sus puntos débiles como aspectos de valores del trabajo en aspectos particulares como en este relato (D-3): "Aquella tesis era aquello que me iba a representar, sabe". El elemento común es que, en la narrativa de las entrevistadas, la palabra academia es el punto de identificación con una identidad profesional de investigadora; y el producto del trabajo la tesis, la disertación o los artículos que fueron o no adecuados a la expectativa de los sujetos, (D-3): "En la academia tu tesis es tu tarjeta de presentación. Por lo tanto, la graduación es resignificada por los graduados en la producción de su narrativa sobre el programa. Se buscó demostrar que el primer elemento o unidad de análisis, en la cuestión de los sentidos del trabajo, es que el trabajo está conectado a un sentido vital, el binomio trabajo y vida tiene un valor fundamental y representa un propósito que fue construido a lo largo de una trayectoria en el posgrado.

La entrevistada (D-4) es profesora universitaria y concluyó en 2007 su maestría en el área de eficiencia energética en tecnología; en el doctorado continuó con la línea de tecnología en energías renovables con hidroenergía. Es graduada en Ciencias Económicas, divorciada y no tiene hijos. La palabra que las egresadas utilizan para dar sentido al trabajo en el posgrado puede diferenciarse en la amplitud y profundidad que la condición de mujer tiene, al recurrir al desarrollo de una carrera académica en el área de la tecnología (D-4): "Yo hice la maestría 
en el área de eficiencia energética en tecnología, y en el doctorado seguí en la línea de tecnología”. El sentido subjetivo que la formación en el posgrado adquiere se relaciona con elementos de la identidad adquiridos por la vivencia en el programa, como en este relato (D-4): "Fue muy buena, fue muy tranquila". Sin embargo, la identidad de profesional de la academia está muy relacionada en todas las narrativas con el concepto de producción, en el sentido de que el trabajo académico tiene que tener visibilidad, manifestarse como artículos y publicaciones, como (D-4): - "Yo produje, pero no tanto como me habría gustado, porque trabajaba y estudiaba". Se observan algunos obstáculos para conciliar el estudio y el trabajo.

El sentido de no producir adquiere en la formación discursiva una ambigüedad, por un lado, la formación fue muy buena, por el otro, el desempeño de varias actividades simultáneamente no permitió que produjera más. Como en este pasaje: "No parecía interesante parar mi trayectoria en la enseńanza, que es la cosa que más me gusta hacer en la vida”. En su narrativa, la idea de la elección profesional está atravesada por una identidad de investigadora y de alguien que también revela en el discurso, una conciencia: "La enseñanza para mí no fue una opción, fue una elección, una elección consciente”. La posición de su trayectoria es subjetivamente apropiada con movimientos de diferenciación en el discurso, en los que aparecen los obstáculos para la formación, como (D-4): "No es una trayectoria fácil, yo estaría mintiendo, sería romántica". La centralidad del trabajo y de la formación profesional en la vida de la mujer que opta por la carrera académica siempre remite al referente del producto y de la productividad, de modo que en su narrativa aparecen sugerencias de mejora para el proceso formativo, en (D-4): "Entonces, creo que tal vez esa carga horaria, sería interesante, no sé, de alguna manera, que pudiéramos agregar valor también a lo que se hace fuera de la universidad". En cuanto a aspectos de la formación la vivencia académica es demarcada por la condición singular de participar en proyectos, como (D-4): "Formé parte de un proyecto, sí, cómo lo iba a olvidar, es un proyecto que se llama Mescuba, un programa cuyo objetivo es estimular, a través de proyectos conjuntos el intercambio de docentes e investigadores brasileños y cubanos, es un convenio que el gobierno brasileño tiene con Cuba". En algunos discursos, se presenta una superposición del valor de las actividades desarrolladas en el trabajo de formación sobre el valor de la vida, en otros, una justa posición o aún una suposición del trabajo como elemento que agrega valor a la vida, como se pretendió demostrar.

La entrevistada (M-5) es graduada y máster en Derecho y maestra en la línea de tecnología y sociedad por el programa de postgrado, trabaja como abogada en el sindicato y está cursando doctorado, es soltera y no tiene hijos. Los significados de la educación académica están entrelazados y muestran una relación entre las demandas educativas de la sociedad que corresponden a los intereses de cada uno de los graduados. Como (M-5): "Yo hice derecho, después hice una maestría en derecho en la UFPR, hice una maestría en tecnología y trabajo en la UTFPR". Los elementos de la productividad afectan las trayectorias de las mujeres y en todas las narraciones aparecen como productores de sentidos subjetivos, (M-5): "Yo produje un artículo con mi orientador, así produje, escribí en libros, pero no recuerdo cuántos". Todo el contenido de la experiencia formativa revela la producción de sentidos subjetivos sobre el objeto de investigación, todas las entrevistadas hacen un balance positivo de la graduación obtenida y, además, de la temática de investigación. (M-5): "También estudié algo muy bueno, que es la economía marxista, una teoría de la dependencia sureña en América Latina”. Sobre la influencia de la formación en el modo de ser, el transformarse en investigadora afirma su visión positiva sobre el programa, (M-5): "Mira, me gusta, 
creo que dos cosas principales, una es la interdisciplinaridad (...) y el hecho de que es un programa muy crítico".

Demuestra, como las demás entrevistadas, enunciados que remiten la estructura de la universidad y sus condiciones de estudio; (M-5): La estructura no es rica, pero es suficiente". Por lo tanto, existe un predominio de la evaluación positiva de la formación obtenida y dirigida hacia el trabajo, no obstante, la diversidad de los sujetos revela en cada expresión un carácter singular, (M-5): "Mira, es lo que me gusta hacer en la vida, de las cosas que me gustan hacer es la que más me gusta, ejercer la abogacía también, pero me gusta más creo ser profesora". Ser maestro es, ante todo, una elección subjetiva, en la que las unidades de acciones de significado relacionadas con ser maestro están llenas de elementos simbólicos y de sentido común que se originan en la vida cotidiana.

Como en este otro relato (M-5): "Me gusta la vida académica". Como en las demás entrevistadas, para esta es también complejo conciliar trabajo y estudio (M-5): "Es muy difícil compatibilizar el doctorado con el trabajo". Sobre la supervivencia de las mujeres en los programas, estas apuntan a las relaciones intersubjetivas que pueden proporcionar soporte $o$ algún tipo de apoyo (M-5): "En la academia me sentía [incómoda] con mi orientador, porque él es una persona muy invasiva, mucho de imponer su voluntad sobre los demás, y creo que lo hace más con mujeres que con hombres". En esta narrativa sobre su relato de ser mujer demuestra la posibilidad de que estas relaciones intersubjetivas entre profesor y alumno puedan ser atravesadas por la cuestión del género, (M-5): "Porque ser mujer hace que usted tenga una responsabilidad sobre otras cosas que influencian el tiempo real que usted tiene. La mujer asume otras responsabilidades y eso influye en su tiempo dedicado para investigar. En la mirada hacia, sus trabajos las entrevistadas demuestran un eslabón entre voces, estilos sociales, personajes, autores y creadores de sentido, en la cadena interminable de actos de habla y acciones que preceden y suceden a cada sujeto que precede y sucede la presencia de cada hombre en el mundo en una determinada cultura, como en este relato: "Yo voy a ser profesora universitaria, con certeza, y yo voy a tener un bufete de abogados, tal vez paralelo".

\section{Conclusiones}

En la perspectiva histórica cultural, el ser humano constituye una subjetividad social e individual, son categorías que según González Rey (2005) pueden ser consideradas como subversivas. En primer lugar, porque desnaturalizan la comprensión de lo social, y en segundo lugar porque alejan la lógica de los universales, del justo y del injusto, del bien y del mal. Así el sujeto y su subjetividad son mediados por las relaciones intersubjetivas que se desarrollan en los contextos sociales y culturales de los que forma parte la mujer. Las narrativas de las egresadas apuntan a la formación en el posgrado como elemento esencial en la configuración de una subjetividad de investigadora.

En la formación subjetiva de la investigadora la producción de los artículos representa un elemento significativo puesto en juego en la trayectoria de las mujeres (González Rey, 2005). La cuestión del género es un diferenciador de la formación, en la medida en que representa la asunción de varios otros papeles sociales que repercuten en la falta de tiempo y espacio para los estudios.

Al buscar conciliar el trabajo con los estudios, la falta de recursos económicos y los hijos pueden ser obstáculos en la carrera. Según Valentova et al., (2017), la falta de becas interfiere en tener mayor participación de las mujeres en las universidades y cursando postgrado, así como dificulta a las mujeres llegar a la carrera de docencia. Las condiciones 
sociales, como la situación de casada o soltera, con o sin hijos, son elementos diferenciadores de posiciones sociales que repercuten en las narrativas de las egresadas. No se puede negar la atribución de papeles sociales diferentes según el género y la condición social de las mujeres, así como la necesidad en tener que permanecer trabajando como otro elemento diferenciador en la trayectoria. En la configuración de una subjetividad social algunos papeles como de esposa, investigadora y científico demuestran el enfrentamiento de diferentes obstáculos para desarrollar una trayectoria académica.

Las narrativas demuestran un discurso que representa el vínculo entre lo singular y lo colectivo, entre el espacio público y privado de trabajo en todas las entrevistadas. Los rasgos comunes de identidad son elementos del trabajo, presentes en la actividad de ser profesora, de ser investigadora y de ser mujer. Es cierto, por los resultados obtenidos, que ser mujer y ser una profesional de la educación, no puede ser tomado como prueba ineludible de los efectos de la formación en la subjetividad de la egresada, pero constituye, a pesar del restringido número de entrevistadas, una ilustración que permite corroborar las amarras simbólicas de la formación, tejidas a lo largo de los discursos. El lugar del postgrado está más allá de la vida académica, es decir, al tener en cuenta lo individual y lo social se deben considerar no solo las polémicas políticas, culturales y económicas que reflejan visiones de mundo diversas, sino también el habla como fenómeno que va moldeando la opinión del locutor inmediato. En este sentido, la subjetividad de la mujer en la ciencia pasa por diferentes papeles y narrativas en las que se perciben discursos tejidos sobre una condición social y cultural de ser mujer.

Las narrativas describen sus trayectorias y vivencias de vida como mujeres. Los papeles profesionales de ser una maestra o doctora son construcciones de la realidad ideológicamente asumidos y compartidos en una determinada clase social. Las vivencias en el posgrado reflejan los momentos pasados, historias y memorias sobre experiencias individuales y colectivas, compartidas con las personas pertenecientes a una misma generación. Las experiencias vividas por las entrevistadas están implicadas en la constitución de ellas como mujeres y profesionales de determinados tipos. En el análisis de las narrativas, evidenciamos que las entrevistadas se enfrentaban con un conjunto de "barreras" para seguir la carrera científica: doble jornada de trabajo, maternidad, productividad en investigación, competencia, prejuicio y discriminación de género.

\section{Referencias}

Antunes, R. (2015). Os sentidos do trabalho: ensaio sobre a afirmação e a negação do trabalho. São Paulo, Brasil: Boitempo Editorial.

Bardagi, M., Lassance, M. C. P., Paradiso, Â. C., \& Menezes, I. A. de. (2006). Career choice and insertion in labor market: Perceptions from college seniors. Psicologia Escolar e Educacional, 10(1), 69-82.

Benevides Soares, A., de Almeida Santos, Z., de Andrade, A. C. \& Siqueira de Souza, M. (2017). Expectativas acadêmicas e habilidades sociais na adaptação à Universidade. Ciencias Psicológicas, 11(1), 77 - 88. Recuperado de http://www. redalyc.org/jatsRepo/4595/459551482009/ $\mathrm{html} /$ index.html

Bolzani, V. da S. (2017). Mulheres na ciência: por que ainda somos tão poucas? Ciência e Cultura, 69 (4), 56-59. Recuperado de https://dx.doi.org/ 10.21800/2317-66602017000400017

CAPES. (2016). Coordenação de Aperfeiçoamento do Ensino Superior. Doi: http://www.capes.gov. br/sala-de-imprensa/noticias/ 8787-mulheres-permanecem-como-maioria-na-pos-graduacao-brasileira 
Closs, L. Q., \& Rocha-de-Oliveira, S. (2015). História de vida e trajetórias profissionais: estudo com executivos brasileiros. $R A C-R e-$ vista de Administração Contemporânea, 19(4), 525-543. Recuperado de https://www.redalyc. org/pdf/840/84039759006.pdf

Dias, M. S. D. L., \& Soares, D. H. P. (2012). A escolha profissional no direcionamento da carreira dos universitários. Psicologia: ciência e profissão, 32(2), 272-283. Recuperado de http://www. scielo.br/pdf/pcp/v32n2/v32n2a02

Gil, A. C. (2008). Como elaborar projetos de pesquisa. Sáo Paulo, Brasil: Atlas.

González Rey, L. F. (2002) Epistemologia cualitativa e subjetividade. São Paulo, Brasil: Thomson Learning.

González Rey, F. L. (Org). (2005) Subjetividade, Complexidade e Pesquisa em Psicologia. São Paulo, Brasil: Thomson Learning.

Da Silva, F. F., \& Ribeiro, P. R. C. (2014). Trajetórias de mulheres na ciência: "ser cientista" e "ser mulher". Ciência \& Educação (Bauru), 20(2), 449-466. Recuperado de https://www.redalyc. org/pdf/2510/251031473012.pdf

Madalozzo, R., \& Artes, R. (2017). Escolhas profissionais e impactos no diferencial salarial entre homens e mulheres. Cadernos de Pesquisa, 47(163), 202-221. Recuperado de https:// dialnet.unirioja.es/servlet/articulo?codigo= 5888129

Melo, H. P. D., \& Rodrigues, L. (2018). Pioneiras da ciência no Brasil: uma história contada doze anos depois. Ciência e Cultura, 70(3), 41-47. Recuperado de http://sbpcacervodigital.org. $\mathrm{br} /$ handle/20.500.11832/2928
Montero, M. (2012). Hacer para transformar: el método en la psicología comunitaria. Buenos Aires, Argentina: Paidós.

Ostrovski, C. S., Sousa, C. M. D., \& Raitz, T. R. (2017). Expectativas com a carreira docente: escolha e inserção profissional de estudantes de Pedagogia. Revista Brasileira de Estudos Pedagógicos, 98(248), 31-46. Doi: https:// dx.doi.org/10.24109/2176-6681.rbep. 98i248.2555

Peñaloza, V., Diógenes, C. G., \& Sousa, S. J. A. (2008). Escolha profissional no curso de administraçãa: tendências empreendedoras e gênero. RAM. Revista de Administração Mackenzie, 9(8), 151-167. Recuperado de http://www. redalyc.org/articulo.oa?id=195416658009

Santos, F. B. O., \& Marques, R. D. C. (2015). Egressas da Escola de Enfermagem Carlos Chagas: campos de atuação. 1936-1948. Escola Anna Nery, 19(2), 363-368. Doi: https://dx.doi.org/ 10.5935/1414-8145.20150050

Valentova, J. V., Otta, E., Silva, M. L., \& McElligott, A. G. (2017). Underrepresentation of women in the senior levels of Brazilian science: PeerJ, (5), e4000. Recuperado de https://peerj.com/ articles/4000/

Vygotski, L. S. (2003). A formação social da mente. São Paulo, Brasil: Martins Fontes.

World Economic Forum. (2016). The Global Gender Report. Recuperado de http://www3.weforum. org/docs/GGGR16/WEF_Global_Gender_ Gap_Report_2016.pdf 


\section{Datos de filiación}

Maria Sara de Lima Dias. Post-doctorado en Psicología por la Universidad Autónoma de Barcelona (2016) como becario del CAPES, doctorado en Psicología por la Universidad Federal de Santa Catarina (2009) tiene una maestría (2004) en Psicología de la Infancia y la Adolescencia y una Licenciatura en Psicología por la Universidad Federal de Paraná (1990). Especialista en Pedagogía Social por la Universidad Católica Portuguesa. Trabaja con proyectos que discuten la tecnología y el trabajo, la salud de los trabajadores y la orientación profesional y la planificación de la carrera. Miembro del Comité de Salud Mental de la UTFPR. Profesora del Departamento de Estudios Sociales (DAESO) de la Universidad Tecnológica Federal de Paraná y profesora del Programa de Posgrado en Tecnología y Sociedad - PPGTE, en la línea de investigación Tecnología y Trabajo.

Paula Caldas Brognoli. Graduada de Administración en la Universidad Tecnológica Federal de Paraná (UTFPR). Actualmente estudiante becada del programa PIBIC 2018/2019 (Fomentado por la Fundación Araucária) de Iniciación Científica del proyecto: La Carrera de las mujeres que trabajan en organizaciones/ tecnología, con plan de trabajo: la elección profesional y el perfil de las mujeres en el entorno laboral. Miembro del Instituto Político Por.De.Para Mulheres. 